\title{
Originales
}

\section{Desigualdad de esfuerzo terapéutico en mujeres con infarto agudo de miocardio: 10 años después}

\author{
R. CALVO-EMBUENA ${ }^{a}$, C. GONZÁLEZ-MONTE ${ }^{b}$, J. LATOUR-PÉREZ ${ }^{c}$, J. BENÍTEZ-PAREJO ${ }^{d}$, \\ V. LACUEVA-MOYA ${ }^{a}$, M.J. BROCH-PORCAR ${ }^{a}$, S. FERRANDIS-BADÍA ${ }^{a}$, V. LOPEZ-CAMPS ${ }^{a}$, \\ V. PARRA-RODRÍGUEZa , E. GÓMEZ-MARTÍNEZa ${ }^{a}$ M.Á. GARCÍA-GARCÍAa \\ D. ARIZO-LEÓNa Y GRUPO ARIAM
}
aServicio de Medicina Intensiva. 'bervicio de Medicina Preventiva. Hospital de Sagunto. Valencia. España 'Servicio de Medicina Intensiva. Hospital de Elche. Alicante. España.
IIngeniero en Informática. Hospital Carlos Haya. Málaga. España.

Objetivo. Estudios previos muestran que las mujeres con infarto agudo de miocardio (IAM) reciben menos fibrinólisis que los hombres. El objetivo de este trabajo es analizar si existen actualmente diferencias de tratamiento ligadas al género y comparar los resultados con los obtenidos hace 10 años.

Diseño. Se trata de un estudio descriptivo retrospectivo que compara pacientes con IAM de los estudios Análisis de Retraso en Infarto Agudo de Miocardio (ARIAM) en 2003-2004 y Proyecto de Análisis Epidemiológico de Paciente Crítico (PAEEC) de 1992-1993.

Ámbito. Servicios de Medicina Intensiva de 86 hospitales en el PAEEC y 120 en el ARIAM.

Pacientes. Nueve mil novecientos ochenta y uno incluidos en el ARIAM y 1.668 en el PAEEC.

Resultados. Las mujeres tienen menos probabilidad de recibir fibrinólisis que los hombres (odds ratio $=0,82, p<0,01$ ), independientemente de su edad, antecedentes, procedencia o tamaño del hospital. La probabilidad de fibrinólisis es asimismo menor en ancianos, pacientes procedentes de planta, hospitales de más de mil camas, enfermos con hipertensión arterial, accidente ce-

Correspondencia: Dra. R. Calvo Embuena.

Servicio de Medicina Intensiva.

Hospital de Sagunto.

Avda. Ramón y Cajal, s/n

46520 Sagunto. Valencia. España.

Correo electrónico: calvo_reg@gva.es

Manuscrito aceptado el 23-I-2008. rebrovascular, diabetes o enfermedad vascular periférica. La probabilidad es mayor en trasladados desde otro hospital (seguidos por los de Urgencias), en hospitales de menos de 300 camas (seguidos por los de 300-1.000) y si hay historia de cardiopatía isquémica. Comparando los dos periodos, ha aumentado la frecuencia de fibrinólisis en ambos géneros, aunque el incremento ha sido mayor en mujeres.

Conclusiones. Las mujeres con IAM continúan recibiendo menos fibrinólisis, aunque existe un aumento en el número de tratamientos, superior al registrado en los hombres.

PALABRAS CLAVE: diferencia de género, fibrinólisis, infarto agudo de miocardio.

GENDER BIAS IN WOMEN WITH MYOCARDIAL INFARCTION: TEN YEARS AFTER

Objective. Previous studies show that the women with acute myocardial infarction (AMI) receive less fibrinolitic treatment than the men. The objective of this study is to analyze if it exists any difference in fibrinolysis related to gender and to compare the results with those obtained 10 years ago.

Design. Retrospective descriptive study that compare patients with AMI of less than 24 hours of evolution of studies Analysis of Delay in Acute Infarct of Myocardium (ARIAM) in 2003-2004 and Project of Analysis Epidemiologist of Critical Patient (PAEEC) of 1992-1993.

Setting. ICUs from 86 hospitals in Spain that participated in the PAEEC study and 120 ICUs in the ARIAM. 
Patients. We compared data of 9,981 patients including in study ARIAM in 2003-2004 with 1,668 of the PAEEC of 1992-1993.

Results. Women were less likely to receive thrombolytic therapy than men (odds ratio $=0.82$, $p<0.01$ ), after adjusting for age, origin, size of the hospital and antecedents. The probability of fibrynolisis is lower in elderly, patients referred from the general ward, in hospitals of more than 1,000 beds and patients with arterial hypertension, stroke, diabetes or peripheral vascular disease. The probability of fibrinólisis is higher when patient is transferred from another hospital (followed by those of Emergencies Room), in the hospitals by less than 300 beds (followed by those of $300-1,000)$ and when history of prior ischemic heart disease exists. Comparing the two periods, has increased the frequency of fibrynolisis in both genders, although the increment has been greater in the women.

Conclusions. The women with AMI continue receiving less fibrynolisis, although exists an increase in the number of treatments superior to register in the men.

KEY WORDS: gender bias, fibrynolisis, acute myocardial infarction.

\section{INTRODUCCIÓN}

Las desigualdades en el tratamiento del paciente con infarto agudo de miocardio (IAM) relacionadas con el género son conocidas desde los noventa ${ }^{1-6}$. Algunos estudios demuestran que las mujeres con IAM reciben tratamiento fibrinolítico con menor frecuencia que los hombres ${ }^{7-20} \mathrm{o}$ con más retraso ${ }^{11,21-24}$, y que no se cumplen los protocolos del tratamiento fibrinolítico tan estrictamente como se hace con los hombres ${ }^{14}$. Otros investigadores encuentran una menor utilización de procedimientos invasivos en las mujeres con $\mathrm{IAM}^{5,9,25-30}$ o muestran que cuando aquellos se llevan a cabo, la enfermedad está más avanzada y es más grave que en el hombre ${ }^{31}$.

Algunos autores sugieren que es la edad más avanzada de la mujer o su mayor número de comorbilidades los responsables de la menor utilización de fibrinólisis y procedimientos invasivos ${ }^{7,13,28,32-46}$. Sin embargo, también se ha reportado que el menor uso de tratamiento de reperfusión en mujeres mayores de 65 años con IAM, respecto a los hombres de la misma edad, se mantiene a pesar de ajustar para diferentes variables clínicas, entre las que se encuentran las comorbilidades ${ }^{47}$.

El objetivo de este trabajo es analizar si existen diferencias ligadas al género en el tratamiento fibrinolítico del IAM y compararlas con los resultados que obtuvimos hace 10 años, que mostraban que las mujeres recibían menos tratamiento fibrinolítico que los hombres ${ }^{48}$.

\section{PACIENTES Y MÉTODOS}

El trabajo se ha realizado a partir de los datos recogidos durante 2003 y 2004 en 120 hospitales españoles que participaron en el estudio Análisis de Retraso en Infarto Agudo de Miocardio (ARIAM) y reportaron datos de 9.981 pacientes. Estos datos se han comparado con los del estudio Proyecto de Análisis Epidemiológico de Paciente Crítico (PAEEC) realizado en 1992 y 1993 en 95 Unidades de Medicina Intensiva en España sobre 1.668 pacientes. Se ha publicado previamente el diseño del estudio PAEEC $^{49,50}$ y del ARIAM ${ }^{51-53}$. Se seleccionaron los pacientes con IAM de menos de 24 horas de evolución y se excluyeron aquellos que procedían de quirófano. De cada paciente se recogió información sobre: género, edad, antecedentes de diabetes, tabaquismo, activo o no, hipertensión arterial, dislipemia, historia de enfermedad vascular periférica, accidente vascular cerebral o cardiopatía isquémica previa, enfermedad pulmonar obstructiva crónica o cor pulmonale, insuficiencia cardiaca congestiva (según la clasificación de Killip en el ARIAM o la valoración clínica en el PAECC), realización de procedimientos de reperfusión (fibrinólisis o intervención coronaria percutánea [ICP]), existencia en las primeras 24 horas de ventilación mecánica o colocación de marcapasos provisional, mortalidad en la Unidad de Cuidados Intensivos (UCI), procedencia del paciente (Urgencias, planta de hospitalización u otro hospital) y tamaño del hospital (menor de 300 camas, 300-1.000 camas y más de 1.000 camas)

\section{Análisis estadístico}

Se realizó un análisis descriptivo y un análisis bivariable para determinar la asociación cruda entre el género y el resto de las variables anteriormente mencionadas. Las variables se expresan como mediana y rango intercuartil. También se analizó la asociación cruda entre reperfusión (fibrinólisis, ICP, e ICP más fibrinólisis) y las variables anteriormente citadas.

Para estimar la probabilidad de recibir tratamiento fibrinolítico se ajustó un modelo de regresión logística multivariante, seleccionando únicamente a aquellos pacientes del estudio ARIAM con IAM con ST elevado más de $2 \mathrm{~mm}$ y en más de dos derivaciones, que son los casos en los que la indicación electrocardiográfica de fibrinólisis es absoluta.

En el estudio PAEEC no se disponía de esta categorización electrocardiográfica y se analizaron los infartos de forma global.

Para analizar los cambios producidos en hombres y mujeres entre los dos periodos estudiados se utilizó la prueba de Ji cuadrado. Para comprobar si las posibles diferencias entre hombres y mujeres se han modificado en las dos etapas se realizó un análisis estratificado que utilizó la prueba de homogeneidad de Mantel-Haenszel. Se consideraron significativos valores de $\mathrm{p}<0,05$. Los programas estadísticos utilizados han sido EPIDAT 3.1 y SPSS 11.0. 
TABLA 1. Características de los 9.981 pacientes incluidos en el estudio ARIAM en el periodo 2003-2004 de acuerdo con el género

\begin{tabular}{|c|c|c|c|}
\hline & $\begin{array}{l}\text { Hombres } \\
\mathrm{n}=7.450\end{array}$ & $\begin{array}{c}\text { Mujeres } \\
\mathrm{n}=2.531\end{array}$ & $\mathrm{p}$ \\
\hline Edad, años & $64(54-73)$ & $73(64-78)$ & $<0,01$ \\
\hline \multicolumn{4}{|l|}{$\mathrm{ECG}^{\mathrm{a}}$} \\
\hline ST elevado $>2 \mathrm{~mm}$ en $>2$ derivaciones & $4.461(60 \%)$ & $1.311(52 \%)$ & $<0,01$ \\
\hline ST elevado $<2 \mathrm{~mm}$ en $<2$ derivaciones & $1.361(18 \%)$ & $545(22 \%)$ & \\
\hline ST descendido & $1.105(15 \%)$ & $482(19 \%)$ & \\
\hline ECG normal & $512(7 \%)$ & $184(7 \%)$ & \\
\hline \multicolumn{4}{|l|}{ Antecedentes médicos } \\
\hline Ninguno & $415(6 \%)$ & $210(8 \%)$ & $<0,01$ \\
\hline Hipertensión arterial & $3.391(45 \%)$ & $1.693(67 \%)$ & $<0,01$ \\
\hline Dislipemia & $2.598(35 \%)$ & $907(36 \%)$ & 0,38 \\
\hline Diabetes mellitus & $1.875(25 \%)$ & $1.073(42 \%)$ & $<0,01$ \\
\hline Accidente vascular cerebral & $391(5 \%)$ & $175(7 \%)$ & $<0,01$ \\
\hline Enfermedad vascular periférica & $478(6 \%)$ & $77(3 \%)$ & $<0,01$ \\
\hline Cardiopatía isquémica & $623(8 \%)$ & $169(7 \%)$ & $<0,01$ \\
\hline Cor pulmonale & $160(2 \%)$ & $19(1 \%)$ & $<0,01$ \\
\hline Tabaquismo & $3.232(43 \%)$ & $267(10 \%)$ & $<0,01$ \\
\hline Extabaquismo & $1.944(26 \%)$ & $56(2 \%)$ & $<0,01$ \\
\hline \multicolumn{4}{|l|}{ Procedencia } \\
\hline Urgencias del hospital & $6.648(89 \%)$ & $2.269(90 \%)$ & $<0,01$ \\
\hline Otro Servicio del hospital & $249(3 \%)$ & $111(4 \%)$ & \\
\hline Otro hospital & $553(7 \%)$ & $151(6 \%)$ & \\
\hline \multicolumn{4}{|l|}{ Insuficiencia cardiaca $^{b}$} \\
\hline Killip I & $5.789(79 \%)$ & $1.671(67 \%)$ & $<0,01$ \\
\hline Killip II & $870(12 \%)$ & $436(17 \%)$ & \\
\hline Killip III & $391(5 \%)$ & $227(9 \%)$ & \\
\hline Killip IV & $287(4 \%)$ & $158(6 \%)$ & \\
\hline Mortalidad en UCI & $395(5 \%)$ & $253(9 \%)$ & $<0,01$ \\
\hline
\end{tabular}

Datos expresados como mediana y rango intercuartil o número absoluto y porcentaje.

a Falta información en 11 hombres y 9 mujeres. ${ }^{b}$ Falta información en 113 hombres y 39 mujeres.

ECG: electrocardiograma; UCI: Unidad de Cuidados Intensivos.

\section{RESULTADOS}

Las características de los pacientes incluidos en el estudio ARIAM en 2003-2004 según su género se muestran en la tabla 1. La edad, la gravedad de la insuficiencia cardiaca y la mortalidad en la UCI fueron significativamente mayores en las mujeres que en los hombres. Los factores de riesgo cardiovascular, la frecuencia de tabaquismo, extabaquismo y enfermedad vascular periférica fueron más frecuentes en los hombres; en tanto que en las mujeres lo fueron, también significativamente, la existencia de hipertensión arterial y la diabetes. El porcentaje de pacientes que cumplía los criterios electrocardiográficos establecidos para la realización de fibrinólisis fue mayor en el grupo de los hombres $(60 \%$ frente al $52 \%$, con $\mathrm{p}<$ $0,01)$.
En la tabla 2 se muestran los procedimientos y el tratamiento de reperfusión que recibieron los enfermos del estudio ARIAM. El número de pacientes que recibió tratamiento fibrinolítico fue de 5.138 (4.713 únicamente recibieron fibrinólisis y 425 recibieron ICP más fibrinólisis). Entre los 5.772 que cumplían criterios electrocardiográficos para tratamiento fibrinolítico, lo recibieron $4.489(78 \%)$ pacientes $(3.529$ de 4.461 hombres y 960 de 1.311 mujeres; $79 \%$ frente al $73 \%$, diferencia absoluta $5,9 \%$, intervalo de confianza del 95\%: 3,2 a 8,6\%).

En la tabla 3 se muestran las características de los pacientes del estudio ARIAM de 2003-2004 clasificados según el tratamiento recibido para la reperfusión. La existencia de antecedentes clínicos se asoció a una menor frecuencia de reperfusión primaria, ex-

TABLA 2. Procedimientos y tratamientos recibidos por los 9.981 pacientes incluidos en el estudio ARIAM en el periodo 2003-2004 de acuerdo al género

\begin{tabular}{|l|c|c|}
\hline & $\begin{array}{c}\text { Hombres } \\
\mathrm{n}=7.450\end{array}$ & $\mathrm{p}=2.531$ \\
\hline Ventilación mecánica & $262(4 \%)$ & $115(5 \%)$ \\
Catéter de arteria pulmonar & $23(0,3 \%)$ & $4(0,2 \%)$ \\
Marcapasos provisional & $129(2 \%)$ & $50(2 \%)$ \\
Reperfusión primaria & & 0,01 \\
$\quad$ Ninguna & $2.970(40 \%)$ & 0,23 \\
Fibrinólisis & $3.667(49 \%)$ & $1.287(51 \%)$ \\
Intervención coronaria percutánea (ICP) & $461(6 \%)$ & $125(5 \%)$ \\
ICP más fibrinólisis & $352(5 \%)$ & $73(3 \%)$ \\
\hline
\end{tabular}


TABLA 3. Características de los pacientes del ARIAM según la reperfusión primaria recibida

\begin{tabular}{|c|c|c|c|c|c|}
\hline & Ninguno & Fibrinólisis & ICP & $\mathrm{ICP}+$ fibrinólisis & $\mathrm{p}$ \\
\hline Hombre & $2.970(40 \%)$ & $3.667(49 \%)$ & $461(6 \%)$ & $352(5 \%)$ & $<0,01$ \\
\hline Mujer & $1.287(51 \%)$ & $1.046(41 \%)$ & $125(5 \%)$ & $73(3 \%)$ & \\
\hline Edad, años & $70(59-76)$ & $65(55-74)$ & $61(51-71)$ & $59(50-68)$ & $<0,01$ \\
\hline \multicolumn{6}{|l|}{ ECG } \\
\hline$\uparrow \mathrm{ST}>2 \mathrm{~mm}>2$ derivaciones & $890(15 \%)$ & $4.112(71 \%)$ & $393(7 \%)$ & $377(6 \%)$ & $<0,01$ \\
\hline$\uparrow \mathrm{ST} 2 \mathrm{~mm}<2$ derivaciones & $1.239(65 \%)$ & $528(28 \%)$ & $103(5 \%)$ & $36(2 \%)$ & \\
\hline ST descendido & $1.481(93 \%)$ & $37(2 \%)$ & $66(4 \%)$ & $3(0,2 \%)$ & \\
\hline ECG normal & $639(92 \%)$ & $27(4 \%)$ & $23(3 \%)$ & $7(1 \%)$ & \\
\hline Killip I & $3.082(41 \%)$ & $3.663(49 \%)$ & $432(6 \%)$ & $283(4 \%)$ & $<0,01$ \\
\hline Killip II & $601(46 \%)$ & $548(42 \%)$ & $75(6 \%)$ & $82(6 \%)$ & \\
\hline Killip III & $351(57 \%)$ & $205(33 \%)$ & $34(5 \%)$ & $28(4 \%)$ & \\
\hline Killip IV & $147(33 \%)$ & $227(51 \%)$ & $39(9 \%)$ & $32(7 \%)$ & \\
\hline Ningún antecedente médico & $254(41 \%)$ & $324(52 \%)$ & $29(5 \%)$ & $18(3 \%)$ & 0,03 \\
\hline Hipertensión arterial & $2.401(47 \%)$ & $2.216(44 \%)$ & $285(6 \%)$ & $182(4 \%)$ & $<0,01$ \\
\hline Dislipemia & $1.564(45 \%)$ & $1.564(45 \%)$ & $209(6 \%)$ & $168(5 \%)$ & $<0,01$ \\
\hline Diabetes mellitus & $1.444(49 \%)$ & $1.218(41 \%)$ & $180(6 \%)$ & $106(4 \%)$ & $<0,01$ \\
\hline Accidente vascular cerebral & $343(61 \%)$ & $175(31 \%)$ & $36(6 \%)$ & $12(2 \%)$ & $<0,01$ \\
\hline Enfermedad vascular periférica & $326(59 \%)$ & $180(32 \%)$ & $33(6 \%)$ & $16(3 \%)$ & $<0,01$ \\
\hline Cardiopatía isquémica & $277(35 \%)$ & $433(55 \%)$ & $39(5 \%)$ & $43(5 \%)$ & $<0,01$ \\
\hline Cor pulmonale & $113(63 \%)$ & $57(32 \%)$ & $6(3 \%)$ & $3(2 \%)$ & $<0,01$ \\
\hline Tabaquismo & $1.171(33 \%)$ & $1.857(53 \%)$ & $264(7 \%)$ & $207(6 \%)$ & $<0,01$ \\
\hline Mortalidad en UCI & $253(6 \%)$ & $313(7 \%)$ & $38(6 \%)$ & $26(6 \%)$ & 0,5 \\
\hline Procedencia de Urgencias del hospital & $3.772(42 \%)$ & $4.253(48 \%)$ & $513(6 \%)$ & $379(4 \%)$ & $<0,01$ \\
\hline Procedencia de otro Servicio & $226(63 \%)$ & $83(23 \%)$ & $43(12 \%)$ & $8(2 \%)$ & \\
\hline Procedencia de otro hospital & $258(37 \%)$ & $377(54 \%)$ & $30(4 \%)$ & $58(5 \%)$ & \\
\hline Hospital de $<300$ camas & $1.215(45 \%)$ & $1.432(54 \%)$ & $20(0,7 \%)$ & $7(0,3 \%)$ & $<0,01$ \\
\hline Hospital de $300-1.000$ camas & $2.597(42 \%)$ & $2.799(46 \%)$ & $411(7 \%)$ & $309(5 \%)$ & \\
\hline Hospital de $>1.000$ camas & $441(37 \%)$ & $473(40 \%)$ & $155(13 \%)$ & $109(9 \%)$ & \\
\hline
\end{tabular}

ECG: electrocardiograma; ICP: intervención coronaria percutánea; UCI; Unidades de Cuidados Intensivos.

cepto si existía historia de cardiopatía isquémica o de tabaquismo.

En el análisis multivariante sobre utilización de la fibrinólisis en la población del ARIAM de 2003-2004 se incluyeron 5.772 pacientes que cumplían criterios electrocardiográficos para dicho tratamiento (tabla 4). El análisis multivariante muestra que la probabilidad de recibir tratamiento fibrinolítico fue significativamente superior entre los hombres. La edad se asoció de forma inversa con la realización de fibrinólisis.

TABLA 4. Análisis multivariante de los factores asociados al tratamiento fibrinolítico en los 5.772 pacientes del estudio ARIAM de 2003-2004 que cumplían criterios para el mismo

\begin{tabular}{|c|c|c|c|}
\hline Variable & N. ${ }^{\circ}$ total fibrinólisis (\%) & Odds ratio (IC 95\%) & $\mathrm{p}$ \\
\hline \multicolumn{4}{|l|}{ Género } \\
\hline Hombre & $3.529(79 \%)$ & 1 & \\
\hline Mujer & $960(73 \%)$ & $0,82(0,71$ a 0,96$)$ & 0,01 \\
\hline \multicolumn{4}{|l|}{ Edad } \\
\hline 25-56 años & $1.390(82 \%)$ & 1 & \\
\hline 57-66 años & $1.079(81 \%)$ & $1,11(0,92$ a 1,35$)$ & 0,27 \\
\hline 67-72 años & $753(81 \%)$ & $0,84(0,69$ a 1,03$)$ & 0,86 \\
\hline$>72$ años & $1.267(73 \%)$ & $0,76(0,64$ a 0,91$)$ & 0,03 \\
\hline Interacción género-edad & & & 0,31 \\
\hline \multicolumn{4}{|l|}{ Procedencia } \\
\hline Planta & $76(49 \%)$ & 1 & \\
\hline Otro hospital & $360(83 \%)$ & $4,60(3,05$ a 6,93$)$ & $<0,01$ \\
\hline Urgencias & $4.053(78 \%)$ & $3,47(2,49$ a 3,82$)$ & $<0,01$ \\
\hline \multicolumn{4}{|l|}{ Tamaño del hospital } \\
\hline$<300$ camas & $1.230(85 \%)$ & 1 & \\
\hline $300-1.000$ camas & $2.738(77 \%)$ & $0,60(0,51$ a 0,71$)$ & $<0,01$ \\
\hline$>1.000$ camas & $521(69 \%)$ & $0,40(0,32$ a 0,50$)$ & $<0,001$ \\
\hline \multicolumn{4}{|l|}{ Antecedentes médicos } \\
\hline Hipertensión arterial & $2.056(75 \%)$ & $0,85(0,75-0,97)$ & 0,02 \\
\hline Diabetes mellitus & $1.110(73 \%)$ & $0,86(0,74-0,99)$ & 0,03 \\
\hline Accidente vascular cerebral & $160(56 \%)$ & $0,38(0,30-0,49)$ & 0,01 \\
\hline Enfermedad vascular periférica & $179(68 \%)$ & $0,71(0,53-0,94)$ & 0,02 \\
\hline Cardiopatía isquémica crónica & $429(85 \%)$ & $1,42(1,09-1,83)$ & $<0,01$ \\
\hline
\end{tabular}

Se muestran únicamente las variables que resultaron estadísticamente significativas.

IC: intervalo de confianza. 
TABLA 5. Análisis multivariante sobre factores asociados al tratamiento de fibrinólisis en los 1.668 pacientes del estudio PAEEC de 1992-1993 con infarto de miocardio

\begin{tabular}{|c|c|c|c|}
\hline Variable & N. ${ }^{\circ}$ total fibrinólisis (\%) & Odds ratio (IC 95\%) & $\mathrm{p}$ \\
\hline \multicolumn{4}{|l|}{ Género } \\
\hline Hombre & $563(43 \%)$ & 1 & \\
\hline Mujer & $87(24 \%)$ & $0,57(0,43-0,56)$ & 0,02 \\
\hline \multicolumn{4}{|l|}{ Edad } \\
\hline 25-56 años & $216(56 \%)$ & 1 & \\
\hline $57-66$ años & $213(44 \%)$ & $0,72(0,54$ a 0,95$)$ & 0,02 \\
\hline 67-72 años & $137(35 \%)$ & $0,53(0,39$ a 0,71$)$ & $<0,01$ \\
\hline$>72$ años & $84(20 \%)$ & $0,25(0,18$ a 0,35$)$ & $<0,01$ \\
\hline Interacción género-edad & & & $>0,05$ \\
\hline \multicolumn{4}{|l|}{ Procedencia } \\
\hline Planta & $19(15 \%)$ & 1 & \\
\hline Otro hospital & $74(41 \%)$ & $3,66(2,02$ a 6,62$)$ & $<0,01$ \\
\hline Urgencias & $557(41 \%)$ & $3,55(2,11$ a 5,95$)$ & $<0,01$ \\
\hline \multicolumn{4}{|l|}{ Tamaño del hospital } \\
\hline$<300$ camas & $253(42 \%)$ & 1 & \\
\hline $300-1.000$ camas & $315(39 \%)$ & $0,82(0,65$ a 1,02$)$ & 0,09 \\
\hline$>1.000$ camas & $82(32 \%)$ & $0,58(0,42$ a 0,81$)$ & $<0,01$ \\
\hline Insuficiencia cardiaca congestiva & $23(16 \%)$ & $0,47(0,29$ a 0,77$)$ & $<0,01$ \\
\hline EPOC & $23(22 \%)$ & $0,60(0,36$ a 1$)$ & 0,05 \\
\hline \multicolumn{4}{|l|}{ Procedimientos } \\
\hline Catéter arteria pulmonar & $13(16 \%)$ & $0,44(0,23$ a 0,84$)$ & 0,01 \\
\hline Ventilación mecánica & $10(14 \%)$ & $0,40(0,19$ a 0,82$)$ & 0,01 \\
\hline
\end{tabular}

EPOC: enfermedad pulmonar obstructiva crónica; IC: intervalo de confianza.

Los pacientes procedentes de planta del hospital tenían menos probabilidades de recibir fibrinólisis que los que venían de Urgencias o los trasladados desde otro hospital. La probabilidad de fibrinólisis era mayor en los hospitales de menos de 300 camas que en los de mayor tamaño. La existencia de hipertensión arterial, accidente vascular cerebral, diabetes y enfermedad vascular periférica disminuía las probabilidades de fibrinólisis, mientras que la historia de cardiopatía isquémica previa la aumentaba.

En el análisis multivariante sobre utilización de fibrinólisis en los pacientes del estudio PAEEC en 1992-1993 (tabla 5), el cual incluía todos los enfer- mos con infarto de miocardio, independientemente de las características electrocardiográficas, muestra resultados similares a los obtenidos en el análisis de los pacientes del estudio ARIAM de 2003-2004.

Al comparar los pacientes con infarto de miocardio de los años 2003-2004 con los de 1992-1993 hemos estudiado si había diferencias significativas entre los dos periodos de tiempo con independencia del género (tabla 6). Así, se ha encontrado que con el transcurso de los años ha aumentado la frecuencia de fibrinólisis y el antecedente de diabetes y ha disminuido la mortalidad, la colocación de catéteres de arteria pulmonar y la implantación de marcapasos pro-

TABLA 6. Comparación de los pacientes con infarto del estudio ARIAM de 2003-2004 con los del estudio PAEEC de 1992-1993

\begin{tabular}{|c|c|c|c|c|c|c|c|}
\hline \multirow[b]{2}{*}{ Variables } & \multicolumn{3}{|c|}{ 1992-1993 } & \multicolumn{4}{|c|}{ 2003-2004 } \\
\hline & Hombre & Mujer & $\mathrm{RP}$ & Hombre & Mujer & $\mathrm{RP}$ & $\mathrm{p}$ \\
\hline Número de pacientes & 1.303 & 365 & & 7.450 & 2.531 & & \\
\hline Edad (mediana, rango IQ) & $64(55-75)$ & $71(65-77)$ & & $64(54-73)$ & $73(64-78)$ & & \\
\hline \multicolumn{8}{|l|}{ Procedencia } \\
\hline Urgencias & $1.056(81 \%)$ & $298(82 \%)$ & & $6.648(89 \%)$ & $2.269(90 \%)$ & & 0,92 \\
\hline Otro Servicio & $95(7 \%)$ & $30(8 \%)$ & & $249(3 \%)$ & $111(4 \%)$ & & 0,51 \\
\hline Otro hospital & $146(11 \%)$ & $36(10 \%)$ & & $553(7 \%)$ & $151(6 \%)$ & & 0,64 \\
\hline \multicolumn{8}{|l|}{ Número de camas } \\
\hline$<300$ & $462(78 \%)$ & $132(22 \%)$ & & $1.976(74 \%)$ & $698(26 \%)$ & & 0,83 \\
\hline $300-1.000$ & $638(79 \%)$ & $174(21 \%)$ & & $4.567(75 \%)$ & $1.549(25 \%)$ & & 0,71 \\
\hline$>1.000$ & $203(78 \%)$ & $59(23 \%)$ & & $898(76 \%)$ & $280(24 \%)$ & & 0,41 \\
\hline Diabetes mellitus ${ }^{\mathrm{a}}$ & $176(14 \%)$ & $128(35 \%)$ & 2,6 & $1.875(25 \%)$ & $1.073(42 \%)$ & 1,68 & $<0,01$ \\
\hline Ventilación mecánica & $50(4 \%)$ & $19(5 \%)$ & 1,35 & $262(4 \%)$ & $115(5 \%)$ & 1,29 & 0,86 \\
\hline Catéter arteria pulmonar ${ }^{\mathrm{a}}$ & $51(4 \%)$ & $29(8 \%)$ & 2,03 & $23(4 \%)$ & $4(0,2 \%)$ & 0,51 & 0,02 \\
\hline Marcapasos provisional $^{\mathrm{a}}$ & $78(6 \%)$ & $27(7 \%)$ & 1,23 & $129(2 \%)$ & $50(2 \%)$ & 1,14 & 0,77 \\
\hline Fibrinólisis ${ }^{\mathrm{a}}$ & $561(43 \%)$ & $87(24 \%)$ & 0,55 & $3.667(49 \%)$ & $1.046(41 \%)$ & 0,84 & $<0,01$ \\
\hline Mortalidad $^{\mathrm{a}}$ & $107(8 \%)$ & $57(16 \%)$ & 1,9 & $395(5 \%)$ & $235(9 \%)$ & 1,75 & 0,63 \\
\hline
\end{tabular}

p: significación de la prueba de homogeneidad de la razón de prevalencia (RP) mujer/hombre entre periodos.

${ }^{a}$ Diferencia significativa entre periodos de tiempo con independencia del sexo $(\mathrm{p}<0,01)$. 
visionales. Por otro lado, hemos investigado si existían diferencias entre los dos estudios de acuerdo al género de los pacientes, para lo que se han comparado las razones de prevalencia mujer/hombre (RP). Así, con respecto a la fibrinólisis, se observa que en el ARIAM de 2003-2004 la probabilidad de fibrinólisis es un $16 \%$ menor en la mujer que en el hombre $(\mathrm{RP}=0,84)$ y hay una variación significativa respecto a los hallazgos del PAEEC, en el cual dicha probabilidad era un $45 \%$ menor $(\mathrm{RP}=0,55)$. La presencia de diabetes ha aumentado más en los hombres que en las mujeres $(\mathrm{RP}=2,60$ frente a $\mathrm{RP}=1,68)$ de forma significativa $(p<0,01)$. En relación con la mortalidad, no se han observado variaciones significativas en los dos periodos estudiados, con mayor mortalidad en las mujeres en ambos $(\mathrm{RP}=1,90$ frente a $\mathrm{RP}=$ $1,75)$.

\section{DISCUSIÓN}

Las mujeres del estudio reciben menos reperfusión primaria, tanto fibrinólisis como ICP, que los hombres. Cuando se cumplen los criterios electrocardiográficos de tratamiento, se hace fibrinólisis en el $73 \%$ frente al $79 \%$ de los hombres. La menor probabilidad de fibrinólisis se mantiene cuando se ajusta por otras variables. Estas diferencias no pueden atribuirse al tipo de infarto, pues en la regresión del estudio se incluyeron solamente aquellos en los que la indicación electrocardiográfica de fibrinólisis es absoluta (ascenso de ST $\geq 2 \mathrm{~mm}$ y en más de dos derivaciones). Tampoco se explica de forma exclusiva por la edad más avanzada de las mujeres, ni por los antecedentes de hipertensión arterial o accidente vascular cerebral que son más frecuentes en el género femenino, pues el sexo fue una variable independiente en el modelo de regresión. Por el contrario, la existencia de otros antecedentes como historia de cardiopatía isquémica, que es más frecuente en los hombres, se asocia a una mayor probabilidad de tratamiento. Resulta remarcable el hecho de que las mujeres también presentan, significativamente, más casos en los que no se conocen factores de riesgo coronario, lo que apunta a favor de la mayor gravedad del cuadro en las mismas como afirman algunos autores ${ }^{11}$. Podría deducirse, dada esta circunstancia, que el tratamiento del IAM debería ser, por lo menos, tan frecuente como en el hombre.

Existen trabajos ${ }^{13,27}$ que afirman que es en los casos en que la indicación de reperfusión es dudosa, esto es, cuando los candidatos no son ideales, cuando las mujeres reciben menos reperfusión que los hombres. Esto puede deberse a la creencia de que existe un mayor riesgo del tratamiento en estas o a una percepción de mayor beneficio en los hombres o, por último, en casos extremos, supondría que se pasan por alto los síntomas.

Hay que destacar que se ha producido un aumento de tratamientos fibrinolíticos en ambos géneros y que este incremento es superior en la mujer que en el hombre pero sin llegar a alcanzarle y manteniendo las diferencias significativas. En esto coincidimos con otros estudios que también han comparado periodos de tiempo y que han continuado encontrando un menor tratamiento de reperfusión en las mujeres que persiste a lo largo de los años ${ }^{20}$.

Por otro lado, coincidiendo con los hallazgos de otros trabajos ${ }^{54}$, vemos que tanto en la población antigua como en la actual la probabilidad de recibir tratamiento fibrinolítico es mayor en aquellos pacientes que son trasladados de un hospital a otro. Destacamos que la cantidad de traslados a otros hospitales continúa siendo inferior en el género femenino, como podemos ver en la tabla 1, que muestra los datos de todos los pacientes con IAM participantes del estudio ARIAM.

La variable tamaño del hospital también se asocia significativamente a la fibrinólisis y explicaría que en los hospitales pequeños que carecen, generalmente, de Servicio de Hemodinámica se realicen más fibrinólisis y trasladen posteriormente a los pacientes a otros centros mayores, bien porque no existan camas en el propio hospital o bien para continuar tratamiento invasivo. Nuestros resultados no concuerdan con estudios anteriores que concluyen que la probabilidad de recibir tratamiento fibrinolítico es mayor en aquellos hospitales que disponen de Servicio de Hemodinámica $^{55}$.

También resulta significativo el aumento de enfermos diabéticos en la población actual, tanto masculina como femenina, con un mayor incremento en la de los hombres. No obstante, los pacientes diabéticos continúan siendo mujeres en su mayoría.

Con respecto a la colocación de catéter de arteria pulmonar, ha descendido su uso de forma significativa especialmente en las mujeres, lo mismo que la implantación de marcapasos provisional. El aumento de tratamiento fibrinolítico, con la mejoría del pronóstico que conlleva, podría explicar ambos hechos.

Destacamos que la existencia de insuficiencia cardiaca, que es más frecuente en mujeres y que en el periodo antiguo se asociaba con menor probabilidad de fibrinólisis, actualmente no se relaciona con la misma.

La letalidad por IAM en UCI ha descendido significativamente tanto en hombres como en mujeres en el último periodo, pero, aunque este descenso ha sido mayor en el género femenino, la mortalidad continúa siendo superior en las mujeres. El descenso de mortalidad superior en las mujeres podría explicarse por el incremento del tratamiento fibrinolítico.

La fuerza del trabajo radicaría en que refleja el comportamiento de los hospitales españoles en cuanto a las diferencias de tratamiento ligadas al género, no completamente descartadas desde los años noventa. Asimismo, nos permite comparar dos periodos y ver si existe una evolución de las actitudes.

Las limitaciones del estudio son principalmente derivadas de su naturaleza retrospectiva, tanto en lo que respecta al periodo 2003-2004, como al de 19921993, lo cual puede conducir a errores en la recogida de datos. Sin embargo, los criterios de inclusión y la definición de las variables fueron muy homogéneos y las características basales de los pacientes fueron comparables. Queremos añadir que, cuando hemos 
comparado ambos periodos, hemos analizado todos los infartos, sin atender a su clasificación electrocardiográfica, de la que no se dispone en el primer estudio, ya que ello podría inducir un sesgo, pues uno de los grupos estaría compuesto únicamente por pacientes en los que la fibrinólisis tiene indicación absoluta.

Por otro lado, muchas de las diferencias detectadas pueden ser estadísticamente significativas debido al tamaño de la muestra.

Por último, no existen datos acerca del cuadro clínico inicial. Su interés radica en que en la mujer los síntomas pueden ser atípicos y ello podría explicar el menor uso de trombolisis.

Así pues, con respecto al tratamiento fibrinolítico, los resultados obtenidos son superponibles a los que obtuvimos hace 10 años ${ }^{48}$ y demuestran que continúan existiendo diferencias en el tratamiento de pacientes con IAM que están ligadas al género femenino.

\section{AGRADECIMIENTOS}

Queremos agradecer a doña Josefa Zamorano Soler, farmacéutica y delegada de Boehringer, la desinteresada ayuda prestada para la obtención de la bibliografía.

\section{Declaración de conflicto de intereses}

Los autores han declarado no tener ningún conflicto de intereses.

\section{BIBLIOGRAFÍA}

1. Ayanian JZ, Epstein AM. Differences in the use of procedures between women and men hospitalized for coronary heart disease. N Engl J Med. 1991;325:221-5.

2. Steingart RM, Packer M, Hamm P, Coglianese ME, Gersh B, Geltman EM, et al. Sex differences in the management of coronary artery disease. Survival and Ventricular Enlargement Investigators. N Engl J Med. 1991;325:226-30.

3. Maynard C, Althouse R, Cerqueira M, Olsufka M, Kennedy JW. Underutilization of thrombolytic therapy in eligible women with acute myocardial infarction. Am J Cardiol. 1991;68:529-30.

4. Dellborg M, Swedberg K. Acute myocardial infarction: difference in the treatment between men and women. Qual Assur Health Care. 1993;5:261-5.

5. Kostis JB, Wilson AC, O'Dowd K, Gregory P, Chelton S, Cosgrove NM, et al. Sex differences in the management and longterm outcome of acute myocardial infarction. A statewide study. MIDAS Study Group. Myocardial Infarction Data Acquisition System. Circulation. 1994;90:1715-30.

6. Clarke KW, Gray D, Keating NA, Hampton JR. Do women with acute myocardial infarction receive the same treatment as men? BMJ. 1994;309:563-6.

7. Marrugat J, Sala J, Masia R, Pavesi M, Sanz G, Valle V, et al. Mortality differences between men and women following first myocardial infarction. RESCATE Investigators. Recursos Empleados en el Síndrome Coronario Agudo y Tiempo de Espera. JAMA. 1998;280:1405-9.

8. McLaughlin TJ, Soumerai SB, Willison DJ, Gurwitz JH, Borbas C, Guadagnoli E, et al. Adherence to national guidelines for drug treatment of suspected acute myocardial infarction: evidence for undertreatment in women and the elderly. Arch Intern Med. 1996;156:799-805.

9. Wong CC, Froelicher ES, Bacchetti P, Barron HV, Gee L, Selby JV, et al. Influence of gender on cardiovascular mortality in acute myocardial infarction patients with high indication for coronary angiography. Circulation. 1997;96:II-51-7.
10. Barron HV, Bowlby LJ, Breen T, Rogers WJ, Canto JG, Zhang Y, et al. Use of reperfusion therapy for acute myocardial infarction in the United States: data from the National Registry of Myocardial Infarction 2. Circulation. 1998;97:1150-6.

11. Vaccarino V, Parsons L, Every NR, Barron HV, Krumholz HM. Sex-based differences in early mortality after myocardial infarction. National Registry of Myocardial Infarction 2 Participants. N Engl J Med. 1999;341:217-25.

12. Gan SC, Beaver SK, Houck PM, MacLehose RF, Lawson HW, Chan L. Treatment of acute myocardial infarction and 30-day mortality among women and men. N Engl J Med. 2000;343:8-15.

13. Rathore SS, Berger AK, Weinfurt KP, Feinleib M, Oetgen WJ, Gersh BJ, et al. Race, sex, poverty, and the medical treatment of acute myocardial infarction in the elderly. Circulation. 2000; 102:642-8.

14. Vermeer F. Thrombolytic therapy in patients of female gender. Thromb Res. 2001;103 Suppl 1:S101-4.

15. Mahon NG, McKenna CJ, Codd MB, O’Rorke C, McCann HA, Sugrue DD. Gender differences in the management and outcome of acute myocardial infarction in unselected patients in the thrombolytic era. Am J Cardiol. 2000;85:921-6.

16. Heer T, Schiele R, Schneider S, Gitt AK, Wienbergen H, Gottwik M, et al. Gender differences in acute myocardial infarction in the era of reperfusion (the MITRA registry). Am J Cardiol. 2002;89:511-7.

17. Kaplan KL, Fitzpatrick P, Cox C, Shammas NW, Marder VJ. Use of thrombolytic therapy for acute myocardial infarction: effects of gender and age on treatment rates. J Thromb Thrombolysis. 2002;13:21-6.

18. Ancona C, Arca M, Saitto C, Agabiti N, Fusco D, Tancioni $\mathrm{V}$, et al. Differences in access to coronary care unit among patients with acute myocardial infarction in Rome: old, ill, and poor people hold the burden of inefficiency. BMC Health Serv Res. 2004;4:34.

19. Theres H, Maier B, Matteucci Gothe R, Schnippa S, Kallischnigg G, Schuren KP, et al. Influence of gender on treatment and short-term mortality of patients with acute myocardial infarction in Berlin. Z Kardiol. 2004;93:954-63.

20. Vaccarino V, Rathore SS, Wenger NK, Frederick PD, Abramson JL, Barron HV, et al. Sex and racial differences in the management of acute myocardial infarction, 1994 through 2002. N Engl J Med. 2005;353:671-82.

21. Indications for fibrinolytic therapy in suspected acute myocardial infarction: collaborative overview of early mortality and major morbidity results from all randomised trials of more than 1000 patients. Fibrinolytic Therapy Trialists' (FTT) Collaborative Group. Lancet. 1994;343:311-22.

22. Vacek JL, Handlin LR, Rosamond TL, Beauchamp G. Gender-related differences in reperfusion treatment allocation and outcome for acute myocardial infarction. Am J Cardiol. 1995;76: 226-9.

23. Weaver WD, White HD, Wilcox RG, Aylward PE, Morris D, Guerci A, et al. Comparisons of characteristics and outcomes among women and men with acute myocardial infarction treated with thrombolytic therapy. GUSTO-I investigators. JAMA. 1996; 275:777-82.

24. Cannon CP, Johnson EB, Cermignani M, Scirica BM, Sagarin MJ, Walls RM. Emergency department thrombolysis critical pathway reduces door-to-drug times in acute myocardial infarction. Clin Cardiol. 1999;22:17-20.

25. Rathore SS, Chen J, Wang Y, Radford MJ, Vaccarino V, Krumholz HM. Sex differences in cardiac catheterization: the role of physician gender. JAMA. 2001;286:2849-56.

26. Alter DA, Naylor CD, Austin PC, Tu JV. Biology or bias: practice patterns and long-term outcomes for men and women with acute myocardial infarction. J Am Coll Cardiol. 2002;39:1909-16.

27. Rathore SS, Wang Y, Radford MJ, Ordin DL, Krumholz HM. Sex differences in cardiac catheterization after acute myocardial infarction: the role of procedure appropriateness. Ann Intern Med. 2002;137:487-93.

28. Harrold LR, Esteban J, Lessard D, Yarzebski J, Gurwitz JH, Gore JM, et al. Narrowing gender differences in procedure use for acute myocardial infarction: insights from the Worcester heart attack study. J Gen Intern Med. 2003;18:423-31.

29. Anand SS, Xie CC, Mehta S, Franzosi MG, Joyner C, Chrolavicius S, et al. Differences in the management and progno- 
sis of women and men who suffer from acute coronary syndromes. J Am Coll Cardiol. 2005;46:1845-51.

30. Martínez-Selles M, López-Palop R, Pérez-David E, Bueno $\mathrm{H}$. Influence of age on gender differences in the management of acute inferior or posterior myocardial infarction. Chest. 2005;128: 792-7.

31. Aguilar MD, Lazaro P, Fitch K, Luengo S. Gender differences in clinical status at time of coronary revascularisation in Spain. J Epidemiol Community Health. 2002;56:555-9.

32. Yarzebski J, Col N, Pagley P, Savageau J, Gore J, Goldberg R. Gender differences and factors associated with the receipt of thrombolytic therapy in patients with acute myocardial infarction: a community-wide perspective. Am Heart J. 1996;131:43-50.

33. Krumholz HM, Murillo JE, Chen J, Vaccarino V, Radford MJ, Ellerbeck EF, et al. Thrombolytic therapy for eligible elderly patients with acute myocardial infarction. JAMA. 1997;277:1683-8.

34. Chandra NC, Ziegelstein RC, Rogers WJ, Tiefenbrunn AJ, Gore JM, French WJ, et al. Observations of the treatment of women in the United States with myocardial infarction: a report from the National Registry of Myocardial Infarction-I. Arch Intern Med. 1998; 158:981-8.

35. Gottlieb S, Harpaz D, Shotan A, Boyko V, Leor J, Cohen M, et al. Sex differences in management and outcome after acute myocardial infarction in the 1990s: a prospective observational community-based study. Israeli Thrombolytic Survey Group. Circulation. 2000;102:2484-90.

36. Raine RA, Black NA, Bowker TJ, Wood DA. Gender differences in the management and outcome of patients with acute coronary artery disease. J Epidemiol Community Health. 2002;56: 791-7.

37. Hanratty B, Lawlor DA, Robinson MB, Sapsford RJ, Greenwood D, Hall A. Sex differences in risk factors, treatment and mortality after acute myocardial infarction: an observational study. J Epidemiol Community Health. 2000;54:912-6.

38. Zubaid M, Rashed WA, Thalib L, Suresh CG. Differences in thrombolytic treatment and in-hospital mortality between women and men after acute myocardial infarction. Jpn Heart J. 2001;42: 669-76.

39. Boucher JM, Racine N, Thanh TH, Rahme E, Brophy J, LeLorier J, et al. Age-related differences in in-hospital mortality and the use of thrombolytic therapy for acute myocardial infarction. CMAJ. 2001;164:1285-90.

40. Harrold LR, Lessard D, Yarzebski J, Gurwitz JH, Gore JM, Goldberg RJ. Age and sex differences in the treatment of patients with initial acute myocardial infarction: a community-wide perspective. Cardiology. 2003;99:39-46.

41. Ali O, Sadiq I, Goldberg RJ, Yarzebski J, Lessard D, Gurwitz J, et al. Age-specific differences in the use of thrombolytic therapy and hospital outcomes in patients with acute myocardial infarction: a community-wide perspective. J Thromb Thrombolysis. 2002;14:5-14

42. Williams RI, Fraser AG, West RR. Gender differences in management after acute myocardial infarction: not 'sexism' but a reflection of age at presentation. J Public Health (Oxf). 2004;26: 259-63.

43. Bakler T, Baburin A, Teesalu R, Rahu M. Comparison of management and 30-day mortality of acute myocardial infarction in men versus women in Estonia. Acta Cardiol. 2004;59:275-81.

44. Cohen M, Gensini GF, Maritz F, Gurfinkel EP, Huber K, Timerman A, et al. The role of gender and other factors as predictors of not receiving reperfusion therapy and of outcome in ST segment elevation myocardial infarction. J Thromb Thrombolysis. 2005; 19:155-61.

45. Morris RW, Whincup PH, Papacosta O, Walker M, Thomson A. Inequalities in coronary revascularisation during the 1990s: evidence from the British regional heart study. Heart. 2005;91: 635-40.

46. Moriel M, Behar S, Tzivoni D, Hod H, Boyko V, Gottlieb S. Management and outcomes of elderly women and men with acute coronary syndromes in 2000 and 2002. Arch Intern Med. 2005; 165:1521-6.

47. Perschbacher JM, Reeder GS, Jacobsen SJ, Weston SA, Killian JM, Slobodova A, et al. Evidence-based therapies for myocardial infarction: secular trends and determinants of practice in the community. Mayo Clin Proc. 2004;79:983-91.

48. Calvo Embuena R. Desigualdad de esfuerzo terapéutico en pacientes con infarto agudo de miocardio (Tesis doctoral). Valencia: 1999.

49. Vázquez Mata G, Rivera Fernández R, González Carmona A, Delgado Rodríguez M, Torres Ruiz JM, Raya Pugnaire A, et al Factors related to quality of life 12 months after discharge from an intensive care unit. Crit Care Med. 1992;20:1257-62.

50. Quality of life: a tool for decision-making in the ICU. Spanish Group for the Epidemiological Analysis of Critical Patients (PAEEC). Intensive Care Med. 1994;20:251-2.

51. Torrado González E, Vera Almazán A, García Alcántara A, Álvarez Bueno JM, Rodríguez García JJ. Introducción. Tema monográfico Proyecto ARIAM. Med Intensiva. 1999;23:271-2.

52. Álvarez Bueno M, Vera Almazán A, Rodríguez García JJ, Ferriz Martín JA, García Paredes T, García Alcántara A y grupo ARIAM. Concepto, desarrollo y objetivos. Tema monográfico Proyecto ARIAM. Med Intensiva. 1999;23:273-9.

53. Aguayo de Hoyos E, Reina Toral A, Colmenero Ruiz M, Barranco Ruiz M, Pola Gallego de Guzmán MD, Jiménez Quintana MM y grupo ARIAM. Análisis de los retrasos en el tratamiento del síndrome coronario agudo. Datos del registro ARIAM. Tema monográfico Proyecto ARIAM. Med Intensiva. 1999;23:280-7

54. Gurwitz JH, Goldberg RJ, Malmgren JA, Barron HV, Tiefenbrunn AJ, Frederick PD, et al. Hospital transfer of patients with acute myocardial infarction: the effects of age, race, and insurance type. Am J Med. 2002;112:528-34.

55. Bobbio M, Bergerone $S$, Maggioni AP, Malacrida R, Franzosi MG, Barlera S, et al. Administration of thrombolytic therapy to 17,944 patients with acute myocardial infarction: the GISSI-3 database. Am Heart J. 1998;135:443-8. 